\title{
Diagnostic role and limitations of 2-deoxy-2-(F-18) fluoro- D-Glucose positron emission tomography (FDG-PET) in screening of synchronous cancer of other organs in patients with head and neck cancers
}

\author{
D. Dequanter*
}

\begin{abstract}
Received: October 11, 2016; Accepted: November 26, 2016; Published: June o9, 2017
*Corresponding author: D. Dequanter, CHU Charleroi, Department of Head and Neck Surgery, Rue de Gozée, 706, 6110 Montigny le Tilleul. E.mail: didier.dequanter@telenet.be
\end{abstract}

\begin{abstract}
Aim

Patients with Head and Neck Squamous Cell Carcinoma (HNSCC) often develop synchronous primary cancers. It is important to detect synchronous cancer in HNSCC patients, because it influences treatment selection of primary cancer. The aim of the study was to evaluate the diagnostic role and limitations of pre-treatment 2-deoxy-2- (F-18) Fluoro-D-Glucose Positron Emission Tomography (FDG-PET) in patients with head and neck cancer.
\end{abstract}

\section{Methods}

Retrospectively, 181 head and neck cancer patients were reviewed to evaluate the clinical significance of pre-treatment FDG-PET.

Results

The synchronous primary tumors were lung in ten cases, head and neck in four cases, colon in three cases, bone in three cases, esophagus in two cases, liver in two cases, pancreas in one case, bladder in one case and adrenal gland in one case.

The diagnostic sensitivity of FDG-PET for synchronous primary cancers was as follows: esophagus 16,6 \% (2/ 12), lung 76,9\% (10/13); head and neck 66,6\% (4/6); bone $75 \%(3 / 4)$ colon $21,4 \%$ (3/14); bladder $100 \%(1 / 1)$; thyroid $0 \%(0 / 1)$ and breast $0 \%(0 / 1)$.

\section{Conclusions}

FDG-PET is an important additional tool for detecting synchronous multiple primary cancers because the diagnostic sensitivity of FDGPET in synchronous head and neck cancer, lung cancer and bone metastasis is high. But the diagnostic sensitivity of FDG-PET to detect esophageal cancer is very low and limited the sensitivity for detection of synchronous multiple primary cancers.

Moreover, the rate of incidental colonic foci of 2-deoxy-2- (F-18) fluoro-D-glucose uptake on FDG-PET was very high.

Key-words: Synchronous multiple primary cancers; FDG-PET sensitivity; head and neck cancer

\section{Introduction}

The occurrence of multiple primary cancers in patients with primary head and neck cancer is well-known phenomenon [1]. It is important to detect synchronous primary cancer in Head and Neck Squamous Cell Carcinoma (HNSCC) patients, because it influences treatment selection of primary head and neck cancer [2].

Particularly, synchronous primary cancer of the respiratory and upper digestive tract is currently the main cause of death after treatment in early stage head and neck cancer patients [3]. For the detection of synchronous primary malignancies before HNSCC treatment, a routine clinical examination is generally performed, followed by fibroscopy and enhanced computed tomography scanning of the head and neck and the thorax [4]. Recently, 2-deoxy-2-(F-18) Fluoro-D-Glucose Positron Emission Tomography (FDG-PET) has become a routine procedure for this purpose.

FDG-PET was introduced clinically to localize viable recurrent and residual tumors at an early stage and with greater confidence as compared to computed tomography [5].

The aim of the study was to evaluate the diagnostic role and limitations of pre-treatment 2-deoxy-2- (F-18) fluoro-Dglucose positron emission tomography (FDG-PET) in localization of synchronous primary cancers in patients with primary head and neck cancer.

\section{Methods}

Retrospectively, 181 head and neck cancer patients were reviewed. Of these patients, 129 were men and 52 were women. The median age at diagnosis was 59 years with a range of 44-87 years. No patients received previously treatment for head and neck cancer.

All the patients underwent either FDG-PET. Each patient fasted for at least $4 \mathrm{~h}$ before intravenous administration of approximately 370 MBq of FDG. The PET scans were performed 1 h after FDG injection. Non contrast-enhanced CT was performed 
from the base of the skull to the upper thighs for attenuation correction and diagnosis. Axial CT images were reconstructed using a soft reconstruction kernel with a $3.75-\mathrm{mm}$ slice thickness and a3.27-mm slice thickness to match the PET images. PET was conducted in the two or three dimensional mode for 3 min per bed position, and images were reconstructed using standard vendor-provided reconstruction algorithms that incorporated ordered subset expectation maximization. Attenuation correction of PET images was performed using attenuation data from CT. The full-width half maximum on the scanner was $6 \mathrm{~mm}$. PET/CT parameters were uniform for all preoperative studies.

When the presence of synchronous primary tumors was suspected clinically or radiographically, additional investigations were performed.

Patients with suspected digestive cancer were referred to gastroenterology for flexible endoscopy with biopsy. Patients with suspected lung cancer were referred to pulmonology for further examinations. Moreover, additional distant lesions detected with PET suggesting synchronous primary tumors were further investigated by histological or cytological study.

\section{Results}

Out of 181 patients, 9 patients (4.9\%) had one synchronous primary tumor; 6 patients $(3.3 \%)$ had three types of cancers. One patient had four types of tumors at initial presentation (Bone/head and neck/esophagus/colon).

The second primary tumors were lung in ten cases, head and neck in four cases, colon in three cases, bone in three cases, esophagus in two cases, liver in two cases, pancreas in one case, bladder in one case and adrenal gland in one case (Table 1)

Table 1: Localization and the number of synchronous primary cancers

\begin{tabular}{|c|c|}
\hline Site & Number of sites \\
\hline Esophagus & 2 \\
\hline Thyroid & 0 \\
\hline Lung & 10 \\
\hline Head and neck & 4 \\
\hline Colon & 3 \\
\hline Liver & 2 \\
\hline Bladder & 1 \\
\hline Bone & 3 \\
\hline Breast & 0 \\
\hline Total & 25 \\
\hline
\end{tabular}

The diagnostic sensitivity of FDG-PET for synchronous primary cancers was as follows: esophagus 16,6 \% (2/ 12), lung $76,9 \%$ (10/13); head and neck 66,6 \% (4/6); bone $75 \%(3 / 4)$ colon $20 \%(3 / 15)$; bladder $100 \%(1 / 1)$; thyroid $0 \%(0 / 1)$ and breast $0 \%(0 / 1)$ (Table 2).In our series, incidental colonic foci of 2-deoxy-2- (F-18) fluoro-D-glucose uptake on PET/CT was very high. 15 patients showed colonic focal PET/CT uptake, but the colonic pathologies were not located at the matched lesion based on PET/CT in $12 / 15$ cases.

Table 2: Diagnostic sensitivity of FDG-PET for synchronous primary tumors

\begin{tabular}{|c|c|}
\hline Primary cancers & Sensitivity of FDG-PET \\
\hline Esophagus & $16,6 \%$ \\
\hline Lung & $76,9 \%$ \\
\hline Head and neck & $66,6 \%$ \\
\hline Bone & $75 \%$ \\
\hline Colon & $20 \%$ \\
\hline Bladder & $10 \%$ \\
\hline Thyroid & $0 \%$ \\
\hline Breast & $0 \%$ \\
\hline
\end{tabular}

\section{Discussion}

In our series, incidental colonic foci of 2-deoxy-2- (F-18) fluoro-D-glucose uptake on PET/CT was very high. The factors that influence the level and pattern of uptake of FDG in the colon are still unclear [6].High diffuse uptake hinders the detection of lesions, while intense focal uptake may result in false positives in the interpretation of malignancy by using PET. Some authors reported that intense colonic 2-deoxy-2- (F-18) fluoro-D-glucose uptake with a focal pattern was observed more frequently in patients with constipation. This means that colonic contraction in association with constipation might result in high FDG uptake. In the literature, incidental colonic foci of 2-deoxy-2- (F-18) fluoroD-glucose uptake on PET/CT have been reported in 1, $3-2.7 \%$ of patients. In our series, a showed colonic focal FDG uptake matched with a true-positive lesion in $3 / 15$ cases leading to a sensitivity of $20 \%$ (compared to the rate of $14.8 \%$ reported by Kondo et al). However, Peng et al demonstrated the necessity of colonoscopy when incidental FDG uptake is found on PET/CT imaging. The authors found that false -positive FDG uptake is more commonly observed in the right colon. Moreover, although SUV max value does not necessarily result in malignancies.

Lee et al confirmed, in their studies, that colonic focal (18) F-FDG uptake by oncology patients warrants endoscopic verification. Complete colonic evaluation by colposcopy is required.

When FDG-PET is performed at initial treatment for head and neck cancer, the diagnostic sensitivity for the second and third synchronous primary is hetereogenous.

In their series, Hanamoto et al identified 57 synchronous primary cancers in 53 patients, of which only $33 \%$ were detected with PET. The most common site for the second primary cancer was the esophagus (49\%), followed by stomach (14\%) and head and neck (11\%).

Kondo et al detected synchronous multiple primary cancers in $42 / 230$ patients $(18,2 \%)$ compared to $9,3 \%$ in our series. Several articles reported that the percentage of synchronous multiple primary cancers in head and neck patients is 2.0$18,0 \%(10)$.The diagnostic sensitivity of PET for synchronous 
primary cancers was as follows: esophagus 7,6 \%, lung 66,7 \%, head and neck $75 \%$, colon $0 \%$ and kidney $0 \%$.

We find similarities with a diagnostic sensitivity of 16,6 $\%$ (2/ 12) for esophagus, 76, $9 \%$ (10/13) for lung; 66,6\% (4/6) for head and neck; $75 \%(3 / 4)$ for bone;21,4 \% (3/14) for colon; $100 \%(1 / 1)$ for bladder; $0 \%(0 / 1)$ for thyroid and $0 \%(0 / 1)$ for breast. These findings lead to the same conclusions as reported by Kondo et al.

FDG-PET is an important additional tool in general for detecting synchronous multiple primary cancers but the diagnostic sensitivity of FDG-PET to detect esophageal cancer is very low and limited the sensitivity for detection of synchronous multiple primary cancers.

\section{References}

1. Shons AR, Mc Quarrie DG. Multiple epidermoid carcinomas of the upper aerodigestive tract. Arch Surg. 1985;120(9):1007-1009. doi:10.1001/archsurg.1985.01390330019003.

2. Linz c, Müller-Richter U, Kircher S, Lapa C, Bluemel C. Value of FDG PET/CT in staging of Oral Cancer: four simultaneous primary malignancies. ClinNuclear Medicine. 2015;40(5):455457. doi:10.1097/ RLU.0000000000000734.

3. Kondo N, Tsukuda M, Nishimura G. Diagnostic sensitivity of 18fluorodeoxyglucose positron emission tomography for detecting synchronous multiple primary cancers in head and neck cancer patients. Eur Arch Otorhinolaryngol. 2012;269(5):1503-1507. doi: 10.1007/ s00405-011-1784-7.

4. Hanamoto A, Takenaka Y, Shimosegawa E, Ymamamoto Y, Yoshii T, Nakahara S, et al. Limitation of 2-deoxy-2- F-18 fluoro-D-Glucose positron emission tomography (FDG-PET) to detect early synchronous primary cancer in patients with untreated head and neck squamous cell cancer. Ann Nucl Med. 2013;27(10):880-885. doi: 10.1007/ s12149-013-0765-x.
5. Klayton T, Li T, Yu JQ Keller L, Cheng J, Cohen SJ, et al. The role of qualitative and quantitative analysis of F18-FDG positron emission tomography in predicting pathologic response following chemoradiotherapy in patients with esophageal carcinoma. J Gastrointest Cancer. 2012;43(4):612-618. doi: 10.1007/s12029-012-9412-3.

6. Shim JH, O JH,O HS, Yoo HM, Jeon Hm, Park CH, et al. Clinical significance of incidental colonic 18 F-FDG uptake on PET/CT images in patients with gastric adenocarcinoma. J Gastrointest Surg. 2012;16(10):18471853. doi:10.1007/s11605-012-1941-3.

7. Peng J, He Y, Xu J, Sheng J, Cai S, Zhang Z. Detection of incidental colorectal tumors with 18F-labelled 2-fluoro-2- deoxyglucose positron emission tomography/computed tomography scans: results of a prospective study. Colorectal Dis. 2011;13(11):374-378. doi: 10.1111/j.1463-1318.2011.02727.x.

8. Treglia G, Calcagni ML, Rufini V, Leccisotti L, Meduri GM, Spitilli MG, et al. Clinical significance of incidental focal colorectal (18) F-fluorodeoxyglucose uptake: our experience and a review of the literature. Colorectal dis 2012;14(2): 174-180. doi: 10.1111/j.1463-1318.2011.02588.x.

9. Lee C, Koh SJ, Lee KL, IM JP, Kim SG, Kim JS, et al. Incidental colonic 18F-fluorodeoxyglucose uptake : do we need colposcopy for patients with focal uptake confined to the left-sided colon ?Dig Dis Sci. 2013,58(1);229-235. doi: 10.1007/s10620-012-2333-y.

10. Bold B, Piao Y, Murata Y, Kishino M, Shibuya H. Usefulness of PET/CT for detecting a second primary cancer. after treatment for squamous cell carcinoma of the head and neck. Clin Nucl Med.2008;33(12):831833. doi: 10.1097/RLU.0b013e31818bf27d. 